\title{
The bending of light and the cosmological constant
}

\author{
Kayll Lake \\ Department of Physics, Queen's University, Kingston, Ontario, Canada, K'L 3N6
}

(October 29, 2018)

The bending of light in Kottler space (the Schwarzschild vacuum with cosmological constant) is examined. Unlike the advance of the perihelion, the cosmological constant produces no change in the bending of light. In this note we examine the conditions under which this statement holds.

PACS numbers: 95.30.Sf, 98.80.Hw, 04.20.Jb

\section{INTRODUCTION}

The deflection of light is one of the "classical" tests of general relativity [1]. With the continuing interest in the cosmological constant [2] one would assume that the effect of a cosmological constant on the deflection of light is well known. Certainly the effect of $\Lambda$ on the advance of the perihelion is well known [3]. This appears not to be the case and is the subject of this note.

Gravitational lensing is now a common tool in astrophysics. There are two approaches to gravitational lensing: the thin lens approximation and the exact approach. These differ fundamentally in that in the thin lens approach there is a background / lens split whereas in the exact approach one uses the full null geodesic equations in an exact solution of Einstein's equations. In a cosmological context, even for strong-lensing calculations, some variant of the thin lens approximation is adequate and used exclusively. For the purposes of the present calculation, however, only the exact approach is appropriate. Recent studies of lensing in the Schwarzschild field are available. Virbhadra and Ellis [4] have examined a strong-field version of the thin lens approximation and Newman and coworkers [5] have compared the exact approach with various approximations.

The present note involves exact lensing in Kottler |6] space, the Schwarzschild vacuum with a cosmological constant $(\Lambda)$. In terms of familiar curvature coordinates $(r, \theta, \phi, t)$ the line element is given by

$$
d s^{2}=\frac{d \mathbf{r}^{2}}{f(\mathrm{r})}+\mathrm{r}^{2}\left(d \theta^{2}+\sin ^{2} \theta d \phi^{2}\right)-f(\mathbf{r}) d t^{2},
$$

where

$$
f(r)=1-\frac{2 m}{r}-\frac{\Lambda r^{2}}{3} .
$$

The associated generalization of the Birkhoff theorem is well known [7]. It is interesting to note that the $\Lambda$ generalization of the black hole uniqueness theorems is not known [8]. Geodesically complete forms of the metric (11) along with Penrose - Carter diagrams are now well know [9].

\section{NULL GEODESICS}

The coordinates $(r, \theta, \phi, t)$ are adapted to two Killing vectors and so geodesics of the metric (11) have two constants of motion. The orbits are stably planar and we choose the plane to be $\theta=\pi / 2$. The momentum conjugate to $\phi$ is the orbital angular momentum $l, r^{2} \dot{\phi}=l$, and the momentum conjugate to $t$ is the energy $\gamma, f(\mathrm{r}) \dot{t}=\gamma$ where $=\frac{d}{d \lambda}$ and $\lambda$ is an affine parameter. Since we are interested here only in null geodesics, it is convenient to reparametrize them with $\bar{\lambda} \equiv l \lambda$ so that

$$
\mathrm{r}^{2} \dot{\phi}=1
$$

and

$$
f(\mathrm{r}) \dot{t}=\frac{1}{b}
$$

where $b \equiv \frac{l}{\gamma}$. If $\Lambda=0$ then $b$ is the impact parameter. From (11), (3) and (4) it follows that

$$
\left(\frac{d r}{d \bar{\lambda}}\right)^{2}=\frac{1}{b^{2}}-\frac{f(r)}{r^{2}}
$$

where $r_{\Sigma}^{2} \equiv b^{2} f\left(r_{\Sigma}\right)$ defines the turning points in $r$ [10]. If (11) is considered derived from a source then $r_{\Sigma}$ is the boundary of that source (see below). From (3) and (5) we have

$$
\left(\frac{d u}{d \phi}\right)^{2}=\left(\frac{m}{b}\right)^{2}+\frac{\Lambda m^{2}}{3}-u^{2}+2 u^{3}
$$

where $u \equiv \frac{m}{r}$. Differentiation of (6) with respect to $\phi$ of course eliminates $\Lambda$, but this does not prove that $\Lambda$ has no effect since (包) is the first integral of the motion [11. In the usual way [12 (6) yields, in first order, the deflection angle

$$
\delta=4 \sqrt{\left(\frac{m}{b}\right)^{2}+\frac{\Lambda m^{2}}{3}} .
$$

Consider the cases $\Lambda=0$ and $\Lambda \neq 0$ separately and assume that $r_{\Sigma}$ and $m$ are fixed, assumptions we examine below. With these conditions it is necessary to distinguish two values of $b$ and $\delta$, say $(b, \delta)$ for $\Lambda=0$ and $(\bar{b}, \bar{\delta})$ for $\Lambda \neq 0$. Note that from (耳), since $\delta$ is measurable, if 
$m$ is known, then $b$ is measurable, but $\bar{b}$ is not since we do not know $\Lambda$ a priori. From the definitions of $r_{\Sigma}, b$ and $\bar{b}$ we have

$$
\Lambda=3 \frac{b^{2}-\bar{b}^{2}}{b^{2} \bar{b}^{2}}
$$

so that from $(7)$

$$
\delta=\bar{\delta}
$$

The fact that (9) is an exact relationship follows by rewriting (6) in the form

$$
\left(\frac{d u}{d \phi}\right)^{2}=u_{\Sigma}^{2}-2 u_{\Sigma}^{3}-u^{2}+2 u^{3}
$$

where $u_{\Sigma} \equiv \frac{m}{r_{\Sigma}}$. Since $u_{\Sigma}$ is fixed by our assumptions on $r_{\Sigma}$ and $m$ it follows that (9) is exact. Although (10) can be solved exactly (the solution is recorded in the Appendix) what is of interest here is the effect of $\Lambda$. In the case of a black hole, without considerations beyond null geodesics, $u_{\Sigma}$ has to be considered merely as a parameter $<\frac{1}{3}$. However, if one inquires into the source of (11) a rather more detailed analysis is required as then both $r_{\Sigma}$ and $m$ enter as derived quantities.

\section{SOURCE}

To see how both $r_{\Sigma}$ and $m$ enter as derived quantities, consider the source of the external field (11) to be a non-singular static perfect fluid [13]. The line element in conventional form is (e.g., 114)

$$
d s^{2}=\frac{d r^{2}}{1-\frac{2 M(r)}{r}}+r^{2}\left(d \theta^{2}+\sin (\theta)^{2} d \phi^{2}\right)-e^{2 \Phi(r)} d t^{2}
$$

with the coordinates comoving in the sense that the fluid streamlines are given by $u^{a}=e^{-\Phi(r)} \delta_{t}^{a}$. Note that we have written (11) without $\Lambda$ motivated by the fact that $M(r)$, on a purely geometrical basis, represents the gravitational energy at a primitive level prior to the introduction of Einstein's equations 15. This choice is, however, merely notation.

\section{A. Generalized T-OV equation}

In terms of the perfect fluid decomposition $\left(T_{b}^{a}=\right.$ $\left.(\rho(r)+p(r)) u^{a} u_{b}+p(r) \delta_{b}^{a}-\Lambda \delta_{b}^{a} /(8 \pi)\right)$, solving for $\Phi^{\prime}(r)$ from the $r$-component of the conservation equations and Einstein's equations $\left(\nabla_{a} T_{r}^{a}=0\right.$ and $\left.G_{r}^{r}-8 \pi p(r)+\Lambda=0\right)$ we obtain the generalized Tolman [16] -OppenheimerVolkoff 17 (T-OV) equation

$$
\Phi^{\prime}(r)=\frac{-p^{\prime}(r)}{\rho(r)+p(r)}=\frac{M(r)+4 \pi p(r) r^{3}-\Lambda r^{3} / 2}{r(r-2 M(r))},
$$

where, from the $t$ component of the Einstein equations $\left(G_{t}^{t}=-8 \pi \rho(r)-\Lambda\right)$,

$$
4 \pi \rho(r)+\Lambda / 2=\frac{M^{\prime}(r)}{r^{2}} .
$$

Despite that fact that the T-OV equation has been known for over sixty years, only recently 18$]$ has its mathematical structure been fully appreciated, even for $\Lambda=0$.

\section{B. Junction Conditions}

The junction of a static perfect fluid onto vacuum in spherical symmetry by way of the Darmois - Israel conditions is well understood 14. Here we follow Musgrave and Lake 19]. To summarize, the continuity of the first fundamental form (intrinsic metric) associated with the boundary $(\Sigma)$ ensures that the continuity of $\theta$ and $\phi$ in metrics (11) and (11) is allowed and that the history of the boundary is given by

$$
r_{\Sigma}=r_{\Sigma}
$$

In terms of intrinsic coordinates $(\tau, \theta, \phi)$, the continuity of the extrinsic curvature component $K_{\tau \tau}$ along with the T-OV equation (12) gives

$$
p\left(r_{\Sigma}\right)=0 .
$$

Equation (15) defines $r_{\Sigma}$ and clearly $\Lambda$ plays no role in that definition. The continuity of the extrinsic curvature components $K_{\theta \theta}$ and $K_{\phi \phi}$ give

$$
\frac{M\left(r_{\Sigma}\right)}{r_{\Sigma}}=\frac{m}{r_{\Sigma}}+\Lambda r_{\Sigma}^{2} / 6
$$

It follows from equations 13 and $(16)$ that

$$
m=\int_{0}^{r_{\Sigma}} 4 \pi r^{2} \rho(r) d r
$$

independent of the value of $\Lambda$.

\section{DISCUSSION}

Equations (14) and (15) show that the turning point (at the boundary of the configuration) $r_{\Sigma}=r_{\Sigma}$ is unaffected by $\Lambda$. With equation (17) then we conclude that if the Kottler-Schwarzschild field is considered generated by a source of specified energy density and isotropic pressure $(\rho(r), p(r))$ then $u_{\Sigma}$ is fixed and by virtue of (10) $\Lambda$ has no effect on the bending of light. Clearly, it is the use of $m$ as opposed to $M\left(r_{\Sigma}\right)$ that is crucial here. When we say "the mass" it is $m$, and not $M\left(r_{\Sigma}\right)$, that we refer to. For example, for timelike orbits in the weak field approximation, $m$ is "the mass", not $M\left(r_{\Sigma}\right)$. Indeed, $M\left(r_{\Sigma}\right)$, though invariantly defined, cannot be given without a 
priori knowledge of $\Lambda$. With the choice that $M\left(r_{\Sigma}\right)$ alone specifies the configuration, it follows from equation (16) that $\Lambda$ would alter the bending of light, but only by way of one's choice as to how the Kottler-Schwarzschild field was specified. The results of this paper can be understood in a more general context. For an irrotational null geodesic congruence there are two types of "focusing": Ricci focusing and Weyl focusing [20]. It is well know, and easy to show, that $\Lambda$ has no effect on Ricci focusing. For the case studied here it is easy to see that $\Lambda$ has no effect on the Weyl focusing either since for the metric (11) with (2) $\Lambda$ does not enter the $C_{\alpha \beta}^{\gamma \delta}$ components of the Weyl tensor.

\section{Acknowledgments}

The comments of a referee helped to improve the presentation. This work was supported by a grant from the Natural Sciences and Engineering research Council of Canada.

[1] C. M. Will, Theory and experiment in gravitational physics (Cambridge University Press, 1993).

[2] A "2001" and "2000" search of astro-ph, gr-qc and hepth at arXiv.org for "cosmological AND constant" reveals 617 archived papers at the time of writing.

[3] See, for example, W. Rindler, Essential Relativity, (Springer-Verlag, New York, 1979).

[4] K. S. Virbhadra and G. F. R. Ellis, Phys. Rev. D62 (2000) 084003 astro-ph/9904193.

[5] See, for example, S. Frittelli, T. P. Kling and E. T. Newman, D61 (2000) 064021 (gr-qc/0001037), T. P. Kling, E. T. Newman and A. Perez, D62 (2000) 024025 (Erratum D62 (2000) 109901, details at gr-qc/0003057).

[6] F. Kottler, Annalen Physik 56, 410 (1918). We use geometrical units throughout.

[7] See, for example, W. B. Bonnor in recent Development in General relativity, (Pergamon, New York, 1962).

[8] W. Israel, Phys. Rev. 164, 1776 (1967). For recent work on the uniqueness theorems with cosmological constant see, P. Chrusciel and W. Simon, J.Math.Phys. 42, 1779 (2001) (gr-qc/0004032).

[9] For an early independent construction see K. Lake and R. C. Roeder, Phys. Rev. D 15 3513, (1977).

[10] We assume that turning points exist. This amounts to the assumption that $3 m<\frac{1}{\sqrt{\Lambda}}$ for $\Lambda>0$ and limits the region of consideration to $f(r)>0$ in general.

[11] Compare J. N. Islam, Phys. Lett. A 97, 239 (1983) and W. Freire, V. Bezerra and J. Lima, Gen. Relativ. Gravit., 33, 1407 (2001).

[12] See, for example, S. Weinberg, Gravitation and Cosmology: Principles and Applications of the General Theory of Relativity, (Wiely, New York, 1972).
[13] A more general source changes in no essential way the argument given here.

[14] See, for example, C. W. Misner, K. S. Thorne, and J. A. Wheeler, Gravitation (W. H. Freeman, San Francisco, 1973).

[15] See S. Hayward, Phys. Rev. D 531938 (1996) grqc/9408002 and E. Poisson and W. Israel, Phys. Rev. D. 41, 1796 (1990).

[16] R. Tolman Relativity Thermodynamics and Cosmology (Dover, New York, 1987)

[17] J. Oppenheimer and G. Volkoff, Phys. Rev. 55, 374 (1939).

[18] T. Baumgarte and A. Rendall, Class. Quant. Grav. 10, 327 (1993).

[19] P. Musgrave and K. Lake, Class. Quant. Grav. 13, 1885 (1996) (gr-qc/9510052).

[20] R. K. Sachs, Proc, Roy. Soc. London A 264, 309 (1961).

\section{Appendix}

The exact solution to 10 is given, up to sign, by

$$
\phi(u)=\sqrt{\frac{k^{2}(u)-\Theta}{l(u) B}} F\left(2 \sqrt{\frac{u_{\Sigma}-u}{A}}, \sqrt{\frac{A}{B}}\right),
$$

where,

$$
\begin{gathered}
k(u)=4 u-1+2 u_{\Sigma}, \\
l(u)=-2 u_{\Sigma}^{2}+u_{\Sigma}-2 u_{\Sigma} u+u-2 u^{2}, \\
A=6 u_{\Sigma}-1+\sqrt{\Theta} \\
B=6 u_{\Sigma}-1-\sqrt{\Theta} \\
\Theta=\left(1-2 u_{\Sigma}\right)\left(1+6 u_{\Sigma}\right),
\end{gathered}
$$

and $F$ is the incomplete elliptic integral of the first kind. 